Model Building and Structure Refinement

Paul Emsley, Oxford university

\title{
Using Coot to Model N-linked Carbohydrates
}

Coot [1] is molecular graphics application initially designed for model-building and validation of protein models using X-ray data. Since its release, the focus of development has shifted to include building and validation tools for ligands and protein-ligand complexes. A particular specialization included recently is the task of building models of glycosylation. Recently the start of the art for glycosylation structures and model-building has been reviewed [2] in which Coot's building has been analysed. This presentation will discuss monomer, link, torsion and reference distance restraints and demonstrate how Coot has been updated and can be used for rapid modelbuilding and refinement of N-linked glycosylation.

[1] "Features and Development of Coot" (2010) Paul Emsley, Bernhard Lohkamp, William G. Scott and Kevin Cowtan", "Acta Crystallographica Section D - Biological Crystallography". Volume 66, Pages 486-501.

[2] "Carbohydrate structure: the rocky road to automation" (2017) Jon Agirre, Gideon J Davies, Keith S Wilson, Kevin D Cowtan "Current Opinion in Structural Biology", Volume 44, Pages $39-47$. 